1 Universidade Federal da Bahia (UFBA), Instituto de Saúde Coletiva (ISC) Salvador (BA), Brasil. Orcid: https://orcid. org/0000-0002-30652144

celepaim@hotmail.com

2 Universidade Federal da Bahia (UFBA), Instituto de Saúde Coletiva (ISC) Salvador (BA), Brasil. Orcid: https://orcid. org/0000-0001-73403132

maria.lirangel@gmail.com

\section{Estado da arte dos observatórios em saúde: narrativas sobre análises de políticas e sistemas}

\author{
State of the art of health observatories: narratives on policy and \\ systems analysis \\ Marcele Carneiro Paim $\mathbf{1}$, Maria Ligia Rangel Santos ${ }^{2}$
}

DOI: 10.1590/0103-11042018S225

RESUMO A democratização e a facilidade de publicação da informação, apoiadas nas novas Tecnologias de Informação e Comunicação (TIC), vêm proporcionando o uso crescente de ferramentas designadas 'observatórios' como um dispositivo institucional para a saúde. A expansão da demanda por informações no processo de elaboração e/ou monitoramento de políticas públicas das mais distintas áreas e campos de produção de conhecimento é um fator que vem oportunizando, nas últimas décadas, a utilização dessas ferramentas em muitos países, entre eles, o Brasil. Este artigo tem por objetivo apresentar os resultados de uma revisão de literatura acerca dos observatórios existentes na área de saúde, caracterizando a produção científica sobre experiências brasileiras e internacionais dedicadas à temática da análise de políticas e sistemas. Foram identificados os principais temas de investigação, pesquisadores e resultados de estudos, com o intuito de discutir as funções desses observatórios e possíveis contribuições para as políticas de saúde. Através de busca avançada em bases de dados, foram encontradas 233 publicações. A partir de critérios de inclusão e exclusão, selecionaram-se 40 estudos para análise e categorização. A maioria dos estudos revela que os observatórios apresentaram como função oferecer informações com credibilidade para acompanhamento de políticas de saúde, planejamento estratégico e tomadas de decisão baseadas em evidências. Cabe ressaltar a tendência de potencializar a capacidade da força de trabalho em pesquisa em saúde, de facilitar a gestão dos dados e a disponibilização da informação, bem como o funcionamento como repositório virtual de informações ou, ainda, a organização em rede. Conclui-se com uma reflexão sobre os limites e possibilidades dos observatórios como uma nova ferramenta para políticas de saúde.

PALAVRAS-CHAVE Observatórios de saúde. Tecnologias de Informação e Comunicação. Políticas de saúde. Sistemas de saúde.

ABSTRACT The democratization and ease of publication of information, supported by new Information and Communication Technologies (ICT), has provided the increasing use of tools designated as 'observatories' as an institutional device for health. The expansion of the demand for information in the process of elaboration and/or monitoring of public policies of the most distinct areas and fields of knowledge production has been a factor that has facilitated, in recent decades, the use of these tools in many countries, among them Brazil. This article aims to present 
the results of a review of the literature on existing observatories in the health area, characterizing the scientific production on Brazilian and international experiences dedicated to the analysis of policies and systems. The main research themes, researchers and study results were identified, with the purpose of discussing the functions of these observatories and possible contributions to health policies. Through an advanced search in databases, 233 publications were found. From the inclusion and exclusion criteria, 40 studies were selected for analysis and categorization. Most of the studies show that observatories had the function of providing credible information for follow-up on health policies, strategic planning and evidence-based decision making. It is worth mentioning the tendency to increase the capacity of the workforce in health research, to facilitate the management of data and the availability of information, as well as the functioning as a virtual repository of information or, also, the network organization. It is concluded with a reflection on the limits and possibilities of observatories as a new tool for health policies.

KEYWORDS Health observatories. Information and Communication Technologies. Health policies. Health systems.

\section{Introdução}

Como resultado da convergência e da interatividade proporcionadas pelo advento das novas Tecnologias de Informação e Comunicação (TIC), é possível apontar o uso paulatino de ferramentas designadas observatórios no âmbito das políticas públicas. A crescente demanda por informações no processo de elaboração e/ou monitoramento dessas políticas, nas mais distintas áreas e campos de produção de conhecimento, é um fator que vem oportunizando, nas últimas décadas, a utilização dessas ferramentas em muitos países, entre eles, o Brasil.

$\mathrm{Na}$ área da saúde, o uso crescente de observatórios revela a importância dada ao uso de informações disponíveis para guiar a elaboração de políticas baseadas em evidências, bem como para dar visibilidade à problemática das desigualdades em saúde'.

Os franceses foram pioneiros na criação do primeiro observatório de saúde, em 1974, que unia os campos da saúde e da assistência social, fornecendo informações sobre a política regional de saúde. Nessa década, surgia, no Canadá, uma das bases para movimentos de convergência na conformação de novo paradigma e conceitos fundamentais abordados na Promoção da Saúde, a partir da qual emergia uma nova perspectiva sobre a saúde, preocupada com o meio social, psicológico e físico das populações, que reconhecia os problemas causados pelas influências multissetoriais sobre a saúde pública².

Nesse contexto, nasceram os observatórios de saúde, como organizações capazes de dispor de informações e recursos, tais como bases de dados, departamentos acadêmicos de saúde pública e diversos parceiros conscientes da utilidade de fundamentar as decisões no conhecimento. Os observatórios de saúde pública foram criados na Inglaterra, nos anos 2000, como instâncias auxiliares importantes para o sistema de saúde pública no País, e constituíram uma rede que se estendeu até cobrir todo o Reino Unido e a Irlanda, para troca de experiências e disseminação rápida de métodos e resultados ${ }^{3}$.

No ano de 1999, a partir de estímulo 
da Organização Pan-Americana da Saúde (Opas), iniciou-se em países do continente americano, incluindo o Brasil, a organização da Rede Observatório de Recursos Humanos em Saúde (ROREHS). Mas, a despeito da existência de observatórios nacionais e internacionais na área da saúde, esse ainda se constitui um tema pouco abordado na literatura especializada. Nas Américas e, particularmente, no Brasil, a discussão e a sistematização de reflexões sobre a experiência de observatórios são recentes na literatura acadêmica ${ }^{2}$.

Este artigo é um ponto de partida para o estudo mais amplo, que objetiva analisar as potencialidades e os limites de observatórios nacionais e internacionais de saúde, conectados em rede, na produção e na disseminação de conhecimento, para contribuir com a formulação e o acompanhamento de políticas de saúde. Portanto, enquanto alicerce do referido estudo, parte da pergunta de investigação: 'Quais as principais características da produção científica acerca do tema Observatórios de Saúde no período 20022017 e quais as lacunas da atualidade?'.

Assim, este estudo tem por objetivo mapear e analisar o estado da arte da produção do conhecimento sobre narrativas constituídas acerca de observatórios de saúde, mais especificamente, observatórios dedicados à temática da análise de políticas e sistemas de saúde, considerando experiências brasileiras e internacionais. O objetivo específico é caracterizar a produção científica acerca dessas narrativas, conforme os seguintes aspectos: tipo de produção; idioma; temáticas dos observatórios referidos nas publicações; ano de publicação; pesquisadores (procedência institucional e geográfica); resultados de estudos e identificação dos principais temas de investigação. Espera-se que essa revisão de literatura possa colaborar com reflexões sobre as funções dos observatórios e com possíveis contribuições para as políticas de saúde.

\section{Material e métodos}

A revisão de literatura é uma etapa presente em toda pesquisa, sempre estabelecendo dependência com a questão estudada e com a modalidade de estudo de revisão a ser desenvolvido. Sua natureza é eminentemente de atualização do conhecimento sobre um tema circunscrito e se sustenta em uma busca bibliográfica para responder a uma questão particular. Este estudo contemplou variados tipos de documentos e teve como princípio metodológico uma revisão de literatura, considerando sua contribuição para formular argumentos coerentes ao compor abstrações e sínteses requeridas por trabalhos de pesquisa, bem como identificando a unidade e a diversidade interpretativa presentes no eixo temático investigado ${ }^{4}$.

O ponto de partida para o mapeamento da produção científica implicou, inicialmente, em fevereiro de 2017, a definição dos Descritores de Assunto, baseando-se na análise daqueles disponíveis em artigos previamente estudados sobre a temática dos observatórios, aliados ao recurso ao vocabulário controlado do DeCS (Descritores em Ciências da Saúde) e à estratégia específica de indexação na Scientific Electronic Library Online (SciELO). Os descritores utilizados na identificação das publicações foram 'health observatories', 'health observatory'.

Dessa forma, para selecionar os estudos incluídos nesta pesquisa, aplicaram-se métodos e técnicas da pesquisa quantitativa e qualitativa, através de busca avançada, utilizando-se o descritor nos campos título e resumo, fazendo-se uso do operador booleano OR, no intervalo entre 2002 e 2017, considerando a disponibilidade dos textos. Assim, foram encontradas $220 \mathrm{pu}-$ blicações (artigos, e-book, livros, fóruns, textos, editoriais, relatórios, tese, mostra, informe e simpósio). No quadro 1, encontra-se a distribuição dos estudos conforme as bases de dados. 
Quadro 1. Distribuição dos artigos conforme base de dados

\begin{tabular}{ll}
\hline Base de dados & Quantidade de artigos \\
\hline Biblioteca Virtual de Saúde (BVS) & 62 \\
Literatura Latino-Americana e do Caribe em Ciências da Saúde (Lilacs) & 26 \\
National Library of Medicine (PubMed) & 63 \\
Scientific Electronic Library Online (SciELO): & 69 \\
\hline Total & $\mathbf{2 2 0}$ \\
\hline
\end{tabular}

Fonte: Elaboração própria.

Para a análise em profundidade, foram estabelecidos os seguintes critérios de inclusão: textos completos disponíveis na internet, temáticas dos estudos relacionadas a política de saúde, planejamento e programação em saúde, gestão de sistemas e serviços de saúde, epidemiologia e/ou experiência de observatório em saúde. A inclusão contemplou a produção de conhecimentos sobre observatórios de saúde, ou seja, estudos que tinham como objeto observatórios de saúde (experiências, análises, projetos, propostas). O horizonte dessa busca, portanto, foi identificar narrativas constituídas sobre observatórios de saúde.

Foram excluídos textos não disponíveis na internet e temas que não correspondessem aos mencionados critérios de inclusão, a saber, estudos sobre observatórios cujas temáticas não estabelecessem relação com políticas e/ou sistemas de saúde, ainda que abordassem conteúdos tais como o cuidado, a assistência, doenças e agravos, como, por exemplo, observatórios sobre câncer, doenças cardiovasculares, cuidados paliativos, cuidados para idosos, demência, entre outros.

Embora os descritores tenham sido usados apenas em inglês, não houve restrição do idioma para triagem dos estudos encontrados. Para seleção dos estudos publicados, foram avaliados os títulos e resumos (abstracts) por dois pesquisadores, atendendo aos critérios de inclusão e exclusão definidos anteriormente. Dessa forma, foram excluídas 187 publicações, considerando, também, duplicidade.

Posteriormente, realizou-se a análise qualitativa exploratória e quantitativa de um conjunto de 33 estudos, destacando-se a leitura na íntegra desses selecionados e sua categorização a partir de uma matriz de análise. Essa matriz contemplou: referência, resumo, link para acesso, ano e tipo de publicação, país, idioma, procedência institucional dos autores, temática do observatório, objetivo do estudo e traduções (quando necessário).

Em maio de 2018, foi feita uma nova busca, seguindo os mesmos procedimentos metodológicos da inicial, que foi realizada em fevereiro de 2017, para atualização, haja vista que a busca anterior não havia contemplado 10 meses do ano de 2017. Assim, foram encontradas mais 13 publicações. Destas, 06 foram excluídas, utilizando-se os mesmos critérios já mencionados, e 07 foram selecionadas, lidas na íntegra e categorizadas a partir da matriz de análise. Logo, os números finais da busca foram: 233 publicações, 193 exclusões e o total de 40 publicações analisadas.

\section{Resultados e discussões}

\section{Análise bibliométrica da produção científica estudada}

Pode-se constatar um aumento do número de publicações sobre a temática do estudo a partir de 2013, bem como a concentração inicial de publicações sobre observatórios em saúde, com foco em análise de políticas e sistemas de saúde no ano de 2003 (gráfico 
1). Esse período corresponde à constituição da rede de observatórios de saúde pública na Inglaterra, conforme relatado por Wilkinson ${ }^{3}$ no artigo em que descreve as lições aprendidas ao longo dos 14 anos de existência desses observatórios junto ao sistema de saúde da Inglaterra.

Gráfico 1. Distribuição temporal dos estudos publicados entre 2002 e 2017

ESTUDOS PUBLICADOS/ANO

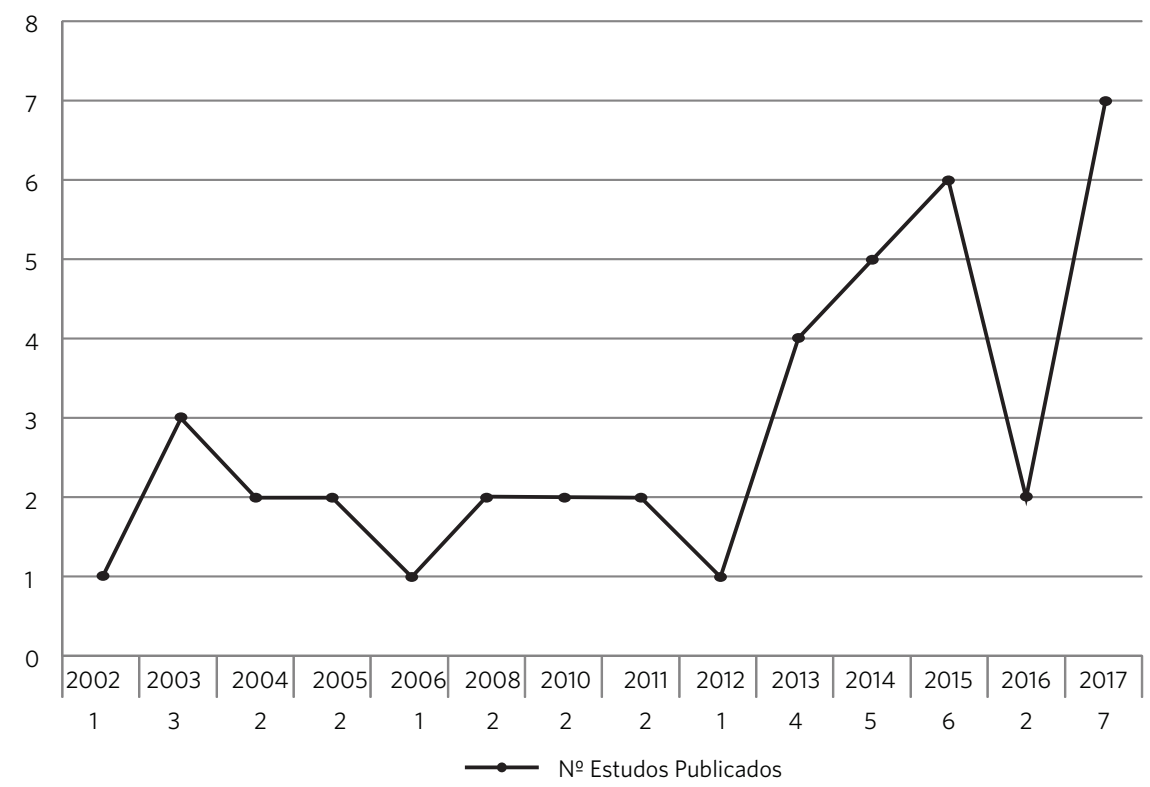

Fonte: Elaboração própria.

Em geral, os estudos tomam como objeto experiências de observatórios de saúde, em diferentes países e estágios de desenvolvimento. Do total das 40 publicações selecionadas, 30 seguem essa linha, o que corresponde a, aproximadamente, $75 \%$. Destes, vale ressaltar que apenas três $\mathbf{2}^{\mathbf{2}, \mathbf{1 3}}$ contemplam também propostas de conceituação acerca dos observatórios de saúde. Outro objeto de estudo que aparece em 09 publicações, isto é, em $22,5 \%$ do total, foi a análise e proposição da criação de novos observatórios. Apenas um estudo5 toma por objeto a produção científica acerca do uso de observatórios em saúde, localizada em bases bibliográficas de dados eletrônicos, através de uma revisão sistemática.

Os achados revelam as primeiras iniciativas de estudos sobre a temática dos observatórios em saúde, e, entre eles, está o artigo de Hemmings e Wilkinson ${ }^{6}$, que busca explicar o que é um observatório de saúde pública e enfoca os observatórios que trabalham com questões de saúde na Europa. O aumento de publicações, a partir de 2013, pode indicar a expansão do uso dos observatórios na área da saúde de forma global, haja vista que a maioria dos objetivos dos estudos se concentra na apresentação de experiências e análise das perspectivas de observatórios de saúde 
pública em vários estágios de desenvolvimento, em diversos países. Merecem destaque, ainda, os estudos que trazem propostas de criação de novos observatórios, publicados, em sua maioria, nos anos mais recentes.

Observa-se, também, a predominância de estudos publicados em inglês, sendo que 22 publicações (55\%) estão nesse idioma. Além desses, foram registrados 13 estudos em português (32,5\%), 04 em espanhol (10\%) e 01 em italiano (2,5\%).

Quanto ao tipo de publicação (gráfico 2), observa-se que a maioria dos estudos analisados (15) é de artigos científicos. Cabe salientar que a única tese de doutorado é de Santos Neto ${ }^{2}$, autor brasileiro que se dedicou a analisar o desenvolvimento da ROREHS e sua produção, discutindo contribuições para a formulação e a tomada de decisão acerca das políticas de recursos humanos do Sistema Único de Saúde (SUS). Ainda que haja uma variedade de tipos de publicação, verifica-se que sete delas só foram registradas uma vez; sendo que, além dos artigos científicos, apenas os livros, textos e fóruns aparecem mais de duas vezes nessa amostragem.

Gráfico 2. Distribuição dos tipos de publicação que compõem a seleção

\section{TIPOS DE PUBLICACÃO}

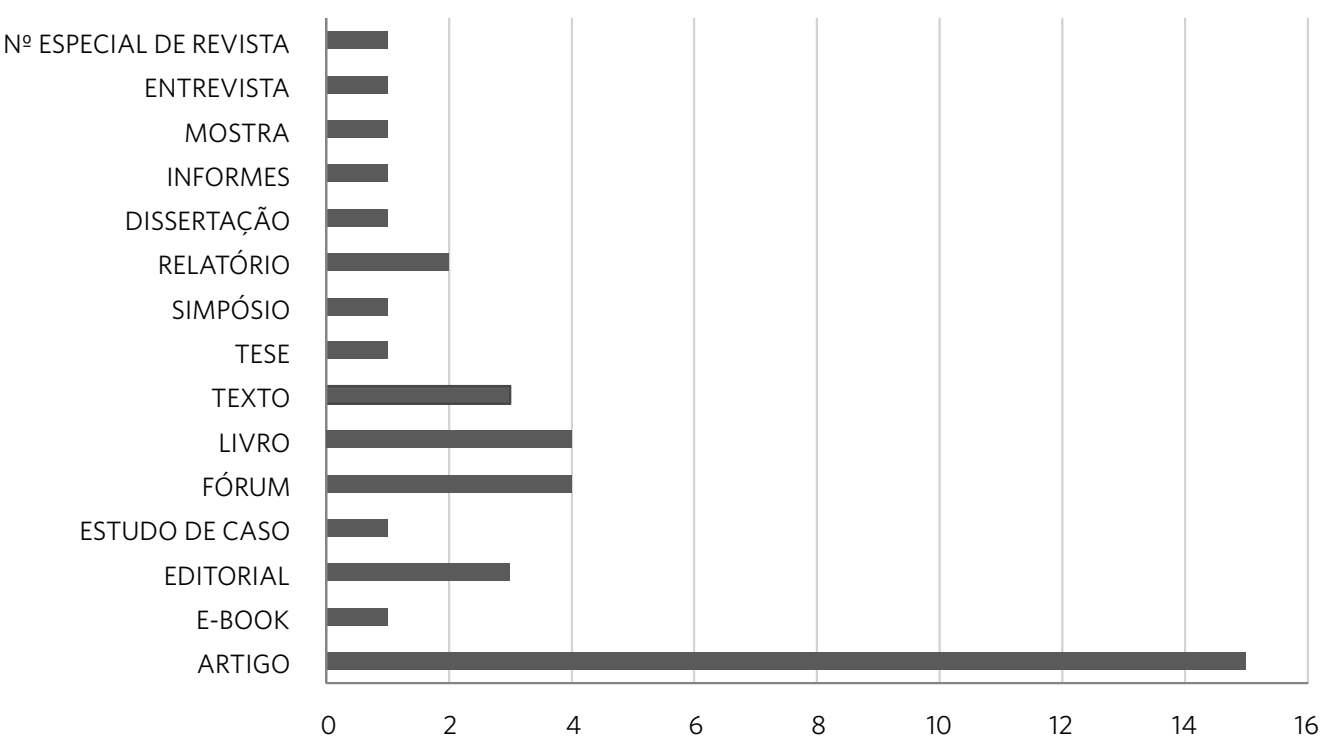

Fonte: Elaboração própria.

Uma visão geral sobre os dados acerca da procedência institucional dos autores revela que o maior quantitativo é de pesquisadores vinculados às universidades, endossando, portanto, a significativa participação dessas instituições nos observatórios de saúde e na produção de conhecimento acerca do tema. Dos 40 estudos, 12 (30\%) não possuem autores procedentes de universidades. Logo, 28 estudos, que correspondem a $70 \%$, têm 
autores de universidades de diversos países. Desses, 21 estudos (75\%) são produções de autoria exclusivamente originada da academia. Os demais 7 estudos, que correspondem a $25 \%$, têm em sua autoria pesquisadores acadêmicos em parceria com pesquisadores de outras instituições governamentais, como Ministérios, Secretarias de Saúde, Institutos Nacionais de Saúde ou, ainda, instituições como Opas e Organização Mundial da Saúde (OMS).

\section{Análise do conteúdo dos documentos}

Quando se observa a distribuição temática dos observatórios que foram objeto de estudo das publicações selecionadas (gráfico 3), destacam-se os temas 'Políticas públicas, gestão e sistemas de saúde' e 'Recursos humanos em saúde', com, respectivamente, 23 e 07 publicações, que, somadas, correspondem a $75 \%$ do total da análise.

Gráfico 3. Distribuição das temáticas dos observatórios que foram objeto dos estudos

\section{TEMÁTICAS DOS OBSERVATÓRIOS}

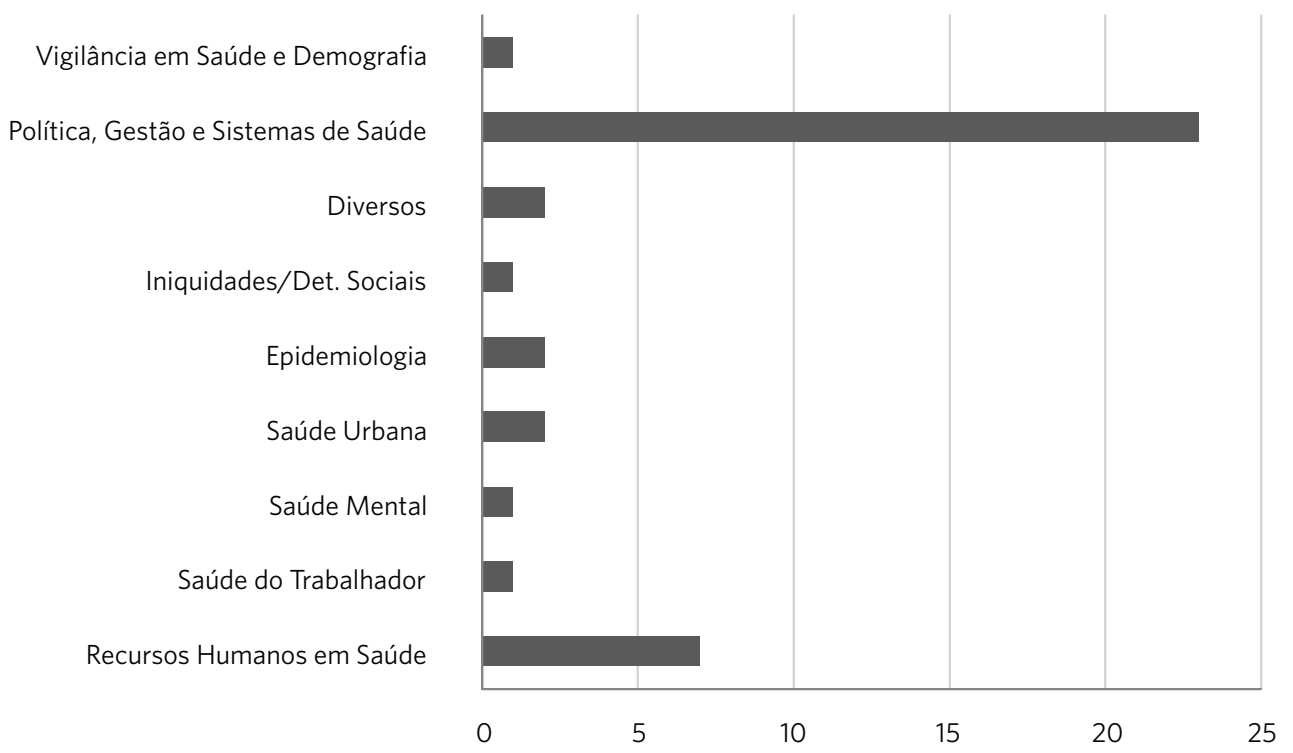

Fonte: Elaboração própria.

No que tange às principais temáticas dos observatórios, cabe destacar alguns achados, conforme descritos a seguir. Dos 23 estudos sobre observatórios centrados no tema 'Políticas públicas, gestão e sistemas de saúde', 16 foram publicados em inglês, $02 \mathrm{em}$ espanhol e 05 em português.
Ainda sobre os estudos acerca dos observatórios centrados no tema 'Políticas públicas, gestão e sistemas de saúde', quanto ao país do observatório estudado, 09 estudos trazem narrativas acerca de observatórios de mais de um país, sendo, portanto, considerados 'híbridos'. Além desses, havia 03 estudos 
sobre observatórios em países do Reino Unido (Inglaterra e Escócia), 02 sobre observatório na Colômbia e 06 sobre observatórios no Brasil, além de Trinidad y Tobago, Estados Unidos da América, Chile, Irã e Malásia, com um estudo cada. Vale ressaltar que os observatórios dos países do Reino Unido fazem parte de todos os estudos considerados híbridos.

A significativa presença de observatórios europeus, especificamente, de países do Reino Unido, bem como dos participantes da Associação de Observatórios de Saúde Pública (APHO) da Inglaterra, aponta para o movimento crescente da utilização dos observatórios na saúde, a partir da União Europeia, com objetivo de apoiar e promover políticas de saúde baseadas em evidências, através de análises da dinâmica dos sistemas de saúde dos países membros.

Sobre os 07 estudos dos observatórios cuja temática é 'Recursos Humanos em Saúde', 04 foram publicados em português, 02 publicados em inglês e 01 em espanhol, sendo que os dois artigos publicados em inglês têm também autores brasileiros. Quanto ao país do observatório estudado, 04 são sobre brasileiros e 03 híbridos, encontrando-se o Brasil como parte de todos os híbridos.

Essa concentração de publicações acerca de narrativas de observatórios com o tema dos Recursos Humanos em Saúde, em vários países da América, incluindo o Brasil, reflete as iniciativas da Opas, bem como do governo brasileiro, para o estímulo de produções na área, a partir da criação da ROREHS.

O quadro 2 sintetiza o material empírico nuclear deste estudo através da categorização temática dos observatórios e outras informações sobre os respectivos estudos contemplados na investigação.

Quadro 2. Categorização temática dos observatórios x informações sobre os respectivos estudos

\begin{tabular}{|c|c|c|c|c|c|}
\hline TÍTULO DO ESTUDO & AUTOR & ANO & OBJETIVO DO ESTUDO & $\begin{array}{l}\text { PAÍS DO } \\
\text { OBSERV. }\end{array}$ & $\begin{array}{l}\text { TEMÁTICA DO } \\
\text { OBERV. }\end{array}$ \\
\hline $\begin{array}{l}\text { 1. Observatório de recursos } \\
\text { humanos em saúde: estação } \\
\text { saúde, trabalho e cidadania: } \\
\text { histórico e ações. }\end{array}$ & $\begin{array}{l}\text { Ticianel F; Char- } \\
\text { bel S. }\end{array}$ & 2014 & $\begin{array}{l}\text { Descrever a criação e aspectos da implementação da } \\
\text { Estação ObservaRH Saúde, Trabalho e Cidadania do } \\
\text { Observatório de RHS da Universidade Federal do MT, } \\
\text { caracterizando os atores, as ações e iniciativas desenvol- } \\
\text { vidas no período de } 2008 \text { a } 2013 \text {. }\end{array}$ & Brasil & $\begin{array}{l}\text { Recursos Huma- } \\
\text { nos em Saúde } \\
\text { (RHS). }\end{array}$ \\
\hline $\begin{array}{l}\text { 3. Observatórios de Recursos } \\
\text { Humanos no Brasil. Estudos e } \\
\text { Análises. }\end{array}$ & $\begin{array}{l}\text { Barros AFR. } \\
\text { (ORG). }\end{array}$ & 2004 & $\begin{array}{l}\text { Apresentar parte da produção das estações de trabalho } \\
\text { da Rede de Observatórios de RHS. }\end{array}$ & Brasil & $\begin{array}{l}\text { Recursos Huma- } \\
\text { nos em Saúde } \\
\text { (RHS). }\end{array}$ \\
\hline $\begin{array}{l}\text { 5. Barriers and facilitators to } \\
\text { establishing a national public } \\
\text { health observatory. }\end{array}$ & $\begin{array}{l}\text { Pooransingh S; } \\
\text { Misir A; Cameron } \\
\text { C; Jaglal S; Ram- } \\
\text { sewak S; Ramdath } \\
\text { D; Goel V. }\end{array}$ & 2015 & $\begin{array}{l}\text { Determinar o que os atores percebem como barreiras e } \\
\text { facilitadores para criar um observatório de saúde pública } \\
\text { nacional em Trinidad e Tobago, bem como as funções es- } \\
\text { pecíficas de um observatório de saúde pública nacional. }\end{array}$ & $\begin{array}{l}\text { Trinidade } \\
\text { e Tobago }\end{array}$ & $\begin{array}{l}\text { Política, gestão } \\
\text { e sistemas de } \\
\text { saúde. }\end{array}$ \\
\hline
\end{tabular}


Quadro 2. (cont.)

\begin{abstract}
6. Developing an academia-based public health observatory: the new global public health observatory with emphasis on urban health at Johns Hopkins Bloomberg School of Public Health.
\end{abstract}

7. Creating a global observatory for health R\&D.

8. The Eastern Region Public Health Observatory.

9. Mapping of available health research and development data: what's there, what's missing, and what role is there for a global observatory?

10. Improving the view of Scotland's health: the impact of a public health observatory upon health improvement policy, action and monitoring in a devolved nation.

11. The Epidemiologic Observatory and the Epidemiology Service: the Campania Region experience.

12. Through a glass darkly: what should public health observatories be observing?

13. Measuring health inequities in low and middle income countries for the development of observatories on inequities and social determinants of health.

14. Toward a Global eHealth Observatory for Nursing.

\author{
Terry RF; Salm \\ JF Jr; Nannei C: \\ Dye C.
}

Castillo-Salgado C. 2015

(n)

Castilo-Salgado C. 2015

Discute o desenvolvimento de um novo observatório de saúde pública com enfoque urbano, pesquisa operacional e infraestrutura de ensino, sob a égide acadêmica do Departamento de Epidemiologia e do Instituto de Saúde Urbana da Johns Hopkins Bloomberg School of Public Health em Baltimore (EUA). Debate o conceito de um observatório de saúde pública e seu papel no enfrentamento das desigualdades sociais em saúde em contextos urbanos locais.

Wright $\mathrm{K}$.

2014
Health England e oferecer dados de saúde da população,
análise e interpretação para priorização e melhoria dos resultados de saúde.

Røttingen A; Regmi 2013 Propor um conjunto de indicadores para monitorar o S; Eide M; Young AJ; Viergever RF; Ardal C; Guzman J; Edwards D; Matlin SA; Terry RF.

Gordon DS; Fisch- 2010 Fazer uma reflexão sobre as contribuições do Scottish bacher C; Stockton D. Public Health Observatory (ScotPHO) e descrever o impacto a curto prazo da ScotPHO sobre a ação, política e monitoramento da melhoria da saúde.

Triassi M, Parlato A; Lopreiato AS.

Watkins F; Bendel $\mathrm{N}$; Samuel S A; Whitehead $M$.
2003

Discutir a estrutura, a função epidemiológica na avaliação Itália de metas em Saúde Pública e na formulação de políticas, bem como descrever a experiência do Observatório Epidemiológico na Região da Campânia.

2002 Estudo qualitativo para avaliar as necessidades dos usuários locais e produtores de informações de saúde pública e a acessibilidade à inteligência em saúde em um observatório regional de saúde pública inglês. Questionar o que os observatórios de saúde pública deveriam estar observando.

Guerra G; Borde E; 2016 Snyder VNS.

Bartz CC; Hardiker 2015 NR; Coenen A.
Discutir questões metodológicas e conceituais para mensuração das desigualdades em saúde nos países de baixa e média renda. Sugerem uma metodologia de três estágios para a criação de observatórios sobre desigualdades em saúde e determinantes sociais da saúde, a partir da experiência do Observatório Brasileiro de Inequidades em Saúde.

Apresentar uma revisão de observatórios de saúde existentes e propor um novo observatório para enfermagem para apoiar a tomada de decisões de enfermagem e desenvolvimento de políticas.

$\begin{aligned} \text { Escócia } & \text { Política, gestão } \\ & \text { e sistemas de } \\ & \text { saúde. }\end{aligned}$

Estados Política, gestão Unidos da e sistemas de América saúde.

Híbrido Política, gestão e sistemas de saúde.

Política, gestão e sistemas de saúde.

Híbrido Política, gestão e sistemas de saúde.

Epidemiologia.

Inglaterra Política, gestão e sistemas de saúde.

Iniquidades, Determinantes sociais.

Híbrido Enfermagem e desenvolvimento de políticas de saúde (Diversos). 


\section{Quadro 2.(cont.)}

15. Colombian National Health Orjuela CC. Observatory: health knowledge management for informed decision making.

16. Developing a conceptual framework of urban health observatories toward integrating research and evidence in to urban policy for health and health equity.

17. Collaboration between health policy and the European observatory on health systems and policies enables open access to selected policy-relevant articles.

18. Health observatories in Iran.

19. A proposed vision: the transatlantic observatory for meeting global health policy challenges through information and communications technology-enabled solutions (ARGOS)

20. Observatorio de políticas públicas en salud universidad de chile: experiencia local de control social en políticas públicas.

21. Public Health Observatories in England: recent transformations and continuing the legacy.

22. The Observatory of the Américas as a network in environmental and worker health in the Americas.

Kano MJ.

Busse R. González C.

Wilkinson J. valho F.
2014 Apresentar a experiência do Instituto Nacional de Saúde da Colômbia na direção do Observatório Nacional da Saúde. Defende que o conhecimento deve apoiar a tomada de decisões e a implementação de políticas públicas e que o observatório possa gerar a informação confiável e oportuna de que o país precisa para responder adequadamente aos desafios representados pela mudança no perfil epidemiológico, e orientar recursos humanos, físicos e financeiros para as necessidades detectadas no campo da saúde.

Caiaffa WT; Friche 2014

AA; Dias MA;

Meireles AL; Ignacio CF; Prasad A;

Desenvolver uma estrutura conceitual de Observatórios de Saúde Urbana como um mecanismo institucional que pode ajudar a sintetizar evidências e incorporá-lo na formulação de políticas urbanas para a saúde e a equidade na saúde.

Apresentar a experiência do Observatório Europeu dos Sistemas e Políticas de Saúde no apoio e na promoção da elaboração de políticas de saúde baseadas em evidências através de uma análise da dinâmica dos sistemas de cuidados de saúde na Europa e envolvimento com decisores políticos.

Rashidian A; Da- 2013 Apresentar os princípios e passos para formulação do mari $\mathrm{B}$; Larijani $\mathrm{BH}$; Moghadda AV;

Alikhani S; Shadpour K; Khosravi A.

Lorenzi N; Moor

2011 Apresentar a contribuição do projeto ARGOS na criação de um o observatório transatlântico para enfrentar os desafios da política global de saúde através de soluções de tecnologia de informação e comunicação, para desenvolver e promover métodos comuns para responder aos desafios globais de saúde na UE e nos EUA.

Crispi F; Menay J; 2016

Apresentar a experiência do Observatório de Políticas Públicas de Saúde (OPPS) e defender que sua inclusão na estrutura institucional formalizaria o compromisso da Universidade com a formação crítica de futuros profissionais de saúde, a fim de contribuir para o debate nacional em políticas públicas.

Descrever as lições aprendidas ao longo dos 14 anos de existência dos Observatórios de Saúde Pública junto ao sistema de saúde da Inglaterra.

Siqueira CE; Car-

\section{Colômbia Política, gestão e sistemas de saúde.}

Brasil Saúde Urbana.

Híbrido

Política, gestão e sistemas de saúde.

Política, gestão e sistemas de saúde.

Híbrido

Política, gestão e sistemas de saúde.

Chile

Política, gestão e sistemas de saúde.

Híbrido

Política, gestão e sistemas de saúde.

Híbrido Recursos Humanos em Saúde (RHS). 


\section{Quadro 2.(cont.)}

23. Análise da política de recursos humanos em saúde: o caso da rede observatório de recursos humanos em saúde do Brasil.

24. Forum: public health observatories and urban health.

25. Mini symposium Public Health Observatories: Development of public health observatories in the UK, Ireland and Europe.

26. Networking collaboratively: The Brazilian Observatório on Human Resources in Health.

27. RELATÓRIO FINAL: Observatório de Recursos Humanos em Saúde.

28. Observatório de Saúde: uma revisão sistemática. 2006.

29. El papel del Observatorio de Salud Mental y los criterios de calidad asistencial de la AEN en la evaluación y planificación asistencial. Recursos de profesionales en CSM y de dispositivos de atención a pacientes con TMG en España.

30. Observatório de recursos humanos em saúde de São Paulo - ObservaRHSP.

31. Public health genomics: the interface with public health intelligence and the role of public health observatories.

32. Relatório sobre a experiên- Oliveira SAS. cia de observatórios de gestão do SUS.

\author{
Santos Neto PM. \\ Kano M. \\ Wilkinso J; Coy- \\ le E.
} ck V. Saúde. FJ.

Seixas $\mathrm{PH}$; NA.

2012

Analisar o desenvolvimento da Rede Observatório de Recursos Humanos em Saúde do Brasil (Rorehs) e sua produção, discutindo em que medida contribui para a formulação de políticas e na tomada de decisão dos atores envolvidos nas políticas de Recursos Humanos do SUS

Campo FE; Hau-

Organização Pan-Americana da

Novell I Fátima V.

Estéve Z; Jiménez D.'Ângelo; Corrêa

Wilkinson J; Ells LJ; 2010 Pencheon D; Flowers J; Burton $\mathrm{H}$;

Apresentar o trabalho do Observatório de recursos humanos em saúde de São Paulo - ObservaRHSP em uma Mostra de experiências inovadoras na gestão da saúde no estado de São Paulo, realizada em 2007.

Examinar a interface entre o novo campo emergente da genômica em saúde pública e o da inteligência em saúde pública. São descritas as possíveis áreas de integração entre esses campos, sugerindo um futuro papel potencial para os Observatórios de Saúde Pública.

008 Fazer um relato sobre a experiência de observatórios de gestão do Sistema Único de Saúde no Brasil, identificando a sua contribuição para o fortalecimento da gestão descentralizada do SUS.

$\begin{array}{cl}\text { Brasil } & \begin{array}{l}\text { Recursos Huma- } \\ \text { nos em Saúde } \\ \text { (RHS). }\end{array} \\ \text { Híbrido } & \begin{array}{l}\text { Política, gestão } \\ \text { e sistemas de } \\ \text { saúde. }\end{array} \\ \text { Híbrido } \quad \begin{array}{l}\text { Política, gestão } \\ \text { e sistemas de } \\ \text { saúde. }\end{array}\end{array}$

Híbrido Recursos Humanos em Saúde (RHS).

Híbrido Recursos Humanos em Saúde (RHS).

Brasil Produção científica Observatório de Saúde (Diversos)

Espanha Saúde Mental.

Brasil Recursos Humanos em Saúde (RHS).

Híbrido Política, gestão e sistemas de saúde.

Brasil Política, gestão e sistemas de saúde. 
Quadro 2.(cont.)

33. What is a public health observatory?

34. HDSS Profile: The South East Asia Community Observatory Health and Demographic Surveillance System (SEACO HDSS).

35. PET Observatório de Saúde Glória/Cruzeiro/Cristal: uma experiência acadêmica e interprofissional em Porto Alegre, RS

36. Priorización para el análisis de información en salud pública. Red de conocimiento del Observatorio Nacional de Prioritization for public health information analysis in Colombia.Knowledge network at the National Health Observatory.

37. Proposta de um observatório epidemiológico do Sistema Único de Saúde.

38. Análise Política em Saúde: contribuições do OAPS.

39. Entrevista com Jairnilson Paim.

40. EDITORIAL Análise Política em Saúde: contribuições do OAPS.

$$
\text { Hemmings J; }
$$$$
\text { Wilkinson J. }
$$

Partap U; Young $\mathrm{EH}$; Allotey P; Soyiri IN; Jahan N; Komahan K; Devarajan N; Sandhu MS; Reidpath DD.

Paiva LL; Rocha CMF; Pinto LF; Nunes A; Santos DS; Anderle M; Tecchio M, Rocha PF, Hoppe PFT, Litvin AK.

Becerra NC; Guzman NA; Montoya JM;Gina A; Sandoval V; Orjuela CC.

Saldanha RF, Bastos RR, Teixeira MTB, Leite ICG, Campos EMS.

Conjunto de pesquisadores vinculados ao OAPS.

Paim J Entrevistado por:Costal I; Conceição P.

Carmen T; Paim J.
2003

Explicar o que é um observatório de saúde pública e enfocar os observatórios que trabalham com questões de saúde. Apresenta, também, observatórios que se relacionam com outros campos, tais como o desenvolvimento econômico ou riscos ambientais.

2017 Apresentar a experiência do Observatório da Comunidade do Sudeste da Ásia (SEACO), sistema de vigilância sanitária e demográfica, estabelecido em Segamat, Malásia, em 2011, na captura de informações longitudinais detalhadas relacionados à saúde e doença entre indivíduos e famílias, que podem ser usadas para melhorar a experiência de saúde na comunidade local e na população.

2017 Apresentar a experiência do PET Observatório de Saúde, da Gerência Distrital Glória/Cruzeiro/Cristal, com a formação de profissionais da saúde sob a perspectiva da integralidade, na medida em que propicia ao acadêmico da saúde vivências nos cenários de prática da rede do SUS, geradoras de novas aprendizagens e conhecimentos de forma contextualizada, rompendo o modelo tradicional de formação em saúde.

2017 Apresentar a experiência do trabalho de Priorização para análise de informações de saúde pública na Colômbia realizado através da Rede de conhecimento no Observatório Nacional da Saúde.

2017 Propor criação de novo observatório de Epidemiologia para o SUS.

2017 Apresentar a produção científica derivada da execução dos projetos e subprojetos desenvolvidos no OAPS, quer assumam a perspectiva da análise política em saúde, quer recortem políticas específicas.

Apresentar parte da produção acadêmica, a partir da elaboração e implementação do Observatório de Análise Política em Saúde (OAPS), articulando uma Rede de pesquisadores voltada para a produção e disseminação de conhecimento em 11 áreas temáticas, que incluem a análise política em saúde e a análise de políticas específicas.

\author{
Reino \\ Política, gestão \\ e sistemas de \\ saúde. \\ Malásia \\ Vigilância sa- \\ nitária e demo- \\ grafia.
}
Brasil Política, gestão e sistemas de saúde.

Colômbia

Política, gestão e sistemas de saúde.

Brasil Epidemiologia.

Brasil

Política, gestão e sistemas de saúde.

Brasil Política, gestão e sistemas de saúde.

Brasil Política, gestão e sistemas de saúde.

Fonte: Elaboração própria. 
A crescente demanda por informações para promover a elaboração e/ou o monitoramento de políticas públicas, das mais distintas áreas e campos de produção de conhecimento, é um fator que vem oportunizando a utilização da estratégia de observatório. Entretanto, a produção de conhecimentos restrita à dimensão conceitual dos observatórios de saúde parece ainda ser incipiente.

Para Hemmings e Wilkinson ${ }^{6}$, embora haja pouca discussão sobre o que constitui um 'observatório', o termo é bem compreendido, ainda que não exista um consenso claro nos muitos novos contextos em que ele é aplicado.

Wilkinson e Coyle ${ }^{\mathbf{1 3}}$ também alertam para o risco que traz a tendência de rotular um número crescente de instituições de 'observatórios', e identificaram sete possíveis papéis para um observatório de saúde: colocar em evidência questões de saúde; olhar para o futuro e alertar sobre possíveis problemas de saúde pública; reunir informações de diferentes fontes sobre novas maneiras de melhorar a saúde; monitorar tendências em saúde e agravos, destacando para áreas de ação; identificar lacunas de informação em saúde; orientar sobre métodos para avaliação, impacto na saúde; avaliar o progresso de instituições e agências locais na melhoria das condições de saúde e redução de iniquidades.

Importante também realçar o pioneirismo europeu no desenvolvimento da cultura de utilização de observatórios na área da saúde, evidenciado pelo número significativo de estudos, cujos argumentos e objetivos comuns norteiam sua utilização: acompanhar o perfil dos sistemas de saúde, realizando comparações entre cidades com problemas semelhantes; promover o acesso a publicações sobre cuidados com a saúde, disseminando informações sobre dados hospitalares e sua interface com a atenção primária e evitando a duplicação de esforços e recursos. Essa abordagem embasa, também, os estudos que propõem a criação de novos observatórios, inclusive modelos de uso global.
Em geral, a maioria dos estudos apresenta como função dos observatórios de saúde oferecer informações com credibilidade para acompanhamento de políticas de saúde, planejamento estratégico e tomada de decisão baseada em evidências. Cabe ressaltar a tendência de potencializar a capacidade da força de trabalho em pesquisa em saúde, facilitar a gestão dos dados e a disponibilização da informação, bem como o funcionamento como repositório virtual de informações ou, ainda, a organização em rede.

Alguns dos estudos mais recentes voltados para as análises de experiências de observatórios destacam a potência da atuação em rede, favorecendo a troca de experiências e a rápida disseminação de métodos e resultados. Castells ${ }^{14}$ considera que a comunicação em rede está revigorando a democracia, ao compreender que as bases de relações interpessoais que estruturam a sociedade humana se modificarão a partir da articulação da sociedade em redes digitais. Desse modo, novos processos interativos estimulam o vínculo propiciado pela atuação em rede, favorecendo, também, o diálogo e a cooperação entre os pesquisadores, motivando, assim, o aprendizado do ofício da pesquisa ${ }^{3,7}$.

Rangel-Santos et al. ${ }^{8}$ alertam para a evolução do modelo de comunicação tradicional para a comunicação científica eletrônica, que permite uma maior integração dos meios na área da saúde. Essa questão pode ser reforçada por Teixeira e Paim7, quando ressaltam a mudança tecnológica causada pela implantação do site do Observatório de Análises Políticas em Saúde (OAPS) na organização do processo de trabalho da rede de pesquisa, e chamam atenção para o advento de uma nova dimensão temporal, que permite um acesso muito mais rápido aos resultados do trabalho de pesquisa de um conjunto de pesquisadores.

Em consonância com a evolução da comunicação científica eletrônica, cabe acrescentar o conceito de convergência midiática, proposto por Henry Jenkins ${ }^{15}$, para designar $o$ atual processo cultural de construção da 
informação, a partir da influência da internet nas principais mídias. Esse autor compreende, ainda, que, ao viver a cultura da convergência, o mundo vivencia a cultura da participação ativa dos usuários no universo midiático, colaborando para uma maior participação política dos indivíduos na sociedade.

Nessa perspectiva, é importante, também, considerar a ideia de democracia digital ou da internet ${ }^{16}$, que se refere à experiência da internet como instrumento de participação popular e recurso para a intervenção do público na esfera da decisão política, tendo em seus dispositivos, portanto, potenciais contribuições para o incremento das práticas e oportunidades democráticas.

Esta revisão aponta, ainda, que as publicações focadas na questão dos Recursos Humanos em Saúde e na saúde dos trabalhadores dos serviços de saúde corroboram a agenda política incentivada pela Opas, através da ROREHS. De acordo com a Opas, o termo observatório reflete a importância dada ao uso de informações e evidências disponíveis para guiar a elaboração de políticas e planos eficazes, beneficiando as populações quanto ao acesso universal a serviços de saúde de boa qualidade e à satisfação de suas necessidades básicas. Além disso, especificamente sobre a ROREHS, sugere que esta influencia positivamente a manutenção do tema dos recursos humanos na agenda política em períodos de transição governamental2.

Apesar de 'observar' se configurar em um importante componente no trabalho dos observatórios, eles parecem ter potencial para ações pró-ativas e contribuições para elaboração de políticas. Dessa forma, para além de entender o fenômeno como algo passivo, é preciso procurar a dinâmica de sua construção e do desenvolvimento de suas principais características.

Nessa perspectiva, parecem estar alinhados com conceitos atuais de redes, cultura da convergência e democracia digital ${ }^{14-16}$, na medida em que podem agregar um repertório de instrumentos capazes de favorecer a participação, contribuindo para que os cidadãos assumam posturas politicamente ativas. Entretanto, apenas a comunicação on-line e o acesso à informação política propiciada por esses dispositivos não asseguram automaticamente a constituição de cidadãos mais informados e mais ativos ${ }^{\mathbf{1 6}}$.

\section{Considerações finais}

Ultimamente, o uso da palavra 'observatório' vem se tornando mais comum, e a expressão tem ganhado espaço na denominação de grupos de pesquisa sociais, redes de pesquisadores e instituições que visam a acompanhar e colaborar com a gestão de recursos públicos. O termo 'observatório' tem sido aplicado a funções de informação e de inteligência para um grande número de áreas, entre elas, a saúde.

O mapeamento da produção científica sobre narrativas constituídas acerca de observatórios dedicados à temática da análise de políticas e sistemas de saúde concebe-se como uma primeira aproximação com o estado da arte desse objeto, a qual será desdobrada em estudos posteriores que identifiquem as abordagens teóricas utilizadas pelos diversos autores. Faz parte, portanto, de um estudo mais amplo acerca das potencialidades e dos limites de observatórios brasileiros e internacionais de saúde, como uma nova ferramenta para políticas de saúde.

Apesar da significativa produção de conhecimentos originada pelos observatórios de saúde em diversos países e continentes, há escassez de publicações sobre esse objeto, haja vista os mais de 40 anos de sua existência na área da saúde, configurando-se, portanto, em uma lacuna no conhecimento.

A expansão de espaços democráticos de discussão e de influência nas decisões políticas, favorecendo a transparência, a accountability, a participação, o acompanhamento e a avaliação, pode configurar novas potencialidades de observatórios. Ainda que a literatura científica sobre observatórios de 
saúde seja incipiente, em geral, os estudos sinalizam sobre a relevância dessa ferramenta para a elaboração de políticas públicas, como espaço de publicização e de participação no processo político de saúde ${ }^{2,9,10}$.

A despeito da existência de observatórios que contemplam a análise de políticas e sistemas de saúde, é necessário aprofundar o conhecimento sobre a maneira como se estruturam e o que oferecem ao público, de sorte que a revisão da literatura não indicou publicações sobre análises de implantação

\section{Referências}

1. Paim J, Paim M. Desenvolvimento do Observatório de Análise Política em Saúde (OAPS): proposta de contribuição para gestores do SUS. Rev. Consensus. 24:30-37, 2017.

2. Santos Neto PMD. Análise da política de recursos humanos em saúde: o caso da rede observatório de recursos humanos em saúde do Brasil. [tese]. [Recife]: Fundação Oswaldo Cruz; 2012.

3. Wilkinson John. Public Health Observatories in England: recent transformations and continuing the legacy. Cad. Saúde Pública. 2015; 31:269-276.

4. Echer IC. A revisão de literatura na construção do trabalho científico. Rev. gaúch. enferm. 2001 jul; 22(2):5-20.

5. Novelli VF. Observatório de Saúde: uma revisão sistemática. [tese]. [São Paulo]: Secretaria de Estado da Saúde de São Paulo; 2006. 163 p. nem avaliações de processos, produtos ou resultados, de acordo com os modelos utilizados em saúde"1.

\section{Colaboradores}

Paim MC contribuiu para a concepção, interpretação dos dados, elaboração do texto e revisão final do artigo. Santos MLR contribuiu para a revisão crítica do conteúdo e revisão final do artigo.
6. Hemmings J, Wilkinson J. Whats is a public health observatory? Epidemiol. community health. 2003; 57:324-326.

7. Teixeira C, Paim J. Análise Política em Saúde: contribuições do OAPS. Saúde em Debat. 2017; 4(esp3):1744.

8. Rangels ML, Guimarães JM, Belens AS. Comunicação e Saúde: aproximação ao estado da arte da produção científica no campo da saúde. In: Paim JS, Almeida-filho N. Saúde Coletiva Teoria e Prática. Rio de Janeiro: MedBook; 2014. p. 625-637.

9. Siqueira CE, Carvalho F. The observatory of the Americas as a network in environmental and worker health in the Americas. Ciênc Saúde Colet. 2003; 8(4):897-902.

10. Bellini MIB, Angnes DI. Rede Observatório de Recursos Humanos em Saúde/Escola de Saúde Públi- 
ca/Rio Grande do Sul: conhecimentos construídos e possibilidades de futuro [internet]. 2008 [acesso em 2018 mar 25]. Disponível em: http://www.esp. rs.gov.br/observatoriorh/img2//LIVRO\%20PARTE2.pdf.

11. Hartz ZMA. Avaliação em saúde: dos modelos conceituais à prática na análise da implantação de programas. Rio de Janeiro: Fiocruz; 1997.

12. Brasil. Ministério da Saúde. Secretaria de Políticas de Saúde. As Cartas da Promoção da Saúde/Ministério da Saúde, Secretaria de Políticas de Saúde, Projeto Promoção da Saúde. Brasília, DF: Ministério da Saúde, 2002.

13. Wilkinson J, Coyle E. Mini symposium Public Health Observatories: Development of public health ob- servatories in the UK, Ireland and Europe. Pub. Health. 2005; 119(4):227-234.

14. Castells M. A sociedade em rede. São Paulo: A Era da Informação v. 1. São Paulo: Paz e Terra; 2003.

15. Jenkins H. Cultura da convergência. 2. ed. São Paulo: Aleph; 2009.

16. Gomes W. A democracia digital e o problema da participação civil na decisão política. Rev. Front. 2005 set-dez; 7(3):214-222.

Recebido em 15/07/2018

Aprovado em 24/09/2018

Conflito de interesses: inexistente

Suporte financeiro: não houve 